\title{
Laser-based Noble-gas Metastable Excitation Techniques with Application to Atom Trap Trace Analysis
}

\author{
Philip S. Light ${ }^{1}$, Rohan D. Glover ${ }^{2}$, Milad A. Dakka ${ }^{1}$, Robert T. Sang ${ }^{2}$ and Andre N. Luiten ${ }^{1}$ \\ ${ }^{1}$ Institute for Photonics and Advanced Sensing and School of Physical Sciences, University of Adelaide, Adelaide, SA 5005, Australia \\ ${ }^{2}$ Centre for Quantum Dynamics, Griffith University, Nathan, QLD 4111, Australia \\ Author e-mail address: philip.light@adelaide.edu.au
}

\begin{abstract}
Atom-trap trace analysis (ATTA) is an ultra-sensitive technique for measurement of noble-gas radio-nuclide ratios at the $10^{-17}$ level, with application to environmental science. We explore laser-based metastable excitation techniques to improve measurement efficiency, accuracy and speed.

OCIS codes: (020.2649) Strong field laser physics; (300.6410) Spectroscopy, multiphoton; (120.3930) Metrological instrumentation; (020.3320) Laser cooling
\end{abstract}

\section{Introduction}

Atom-trap trace analysis (ATTA) is a laser-based technique for measuring noble-gas radio-nuclide ratios (specifically ${ }^{81} \mathrm{Kr},{ }^{85} \mathrm{Kr}$ and ${ }^{39} \mathrm{Ar}$ ) for the dating of ground-water and ocean-water samples [1]. It allows determination of water ages ranging from less than a year, through to millions of years, by comparing the abundance of the specific radio-nuclide in the sample with the known abundance in 'new' water, which is known to be in equilibrium with the atmospheric abundance. ATTA uses magneto-optical trapping to count individual atoms of the rare radio-isotopes, which have an abundance as low as $10^{-17}$ of the abundance of the stable isotope.

Laser cooling of the noble-gas sample first requires excitation of the atoms into the metastable state, from which laser-cooling is readily achievable using standard diode lasers at $\sim 810 \mathrm{~nm}$. Currently, ATTA systems use RF discharge techniques to excite an atom into the metastable state; however, this discharge is not selective, resulting in poor excitation efficiency ( $\sim 10^{-4}$ of excited atoms in desired state), as well as contamination problems in the vacuum system caused by high kinetic energy ions produced in the discharge process. Finding more efficient and cleaner metastable excitation methods would allow reduced measurement time, reduced flushing time, and allow for measurement of smaller samples.

While optical lamp-based metastable excitation techniques have been considered before [2], laser-based schemes remain largely unexplored. Here we consider two laser-based optical excitation techniques and explore how they could benefit an ATTA measurement system.

\section{Multi-photon Excitation}

We have modeled single, two- and three- photon transitions for optical excitation of the metastable level. As shown in fig. 1, a two-photon excitation into the $5 \mathrm{p}^{2}[3 / 2]_{\mathrm{J}=2}$ states decays directly into the desired metastable state with $77 \%$ branching ratio. For single- and three-photon excitation, selection rules require excitation first into the $5 s^{2}[3 / 2]_{J=1}$, followed by single-photon excitation to the $5 \mathrm{p}^{2}$ state using an additional infrared laser at $819 \mathrm{~nm}$.

We conclude that two-photon excitation is most feasible, as although higher intensity is required than singlephoton excitation, much higher pulse energies are available at the longer wavelength. For a commercially available laser source (Sirah Cobra) with $1 \mathrm{GHz}$ linewidth, we calculate a metastable excitation efficiency of $3 \%$ in the twophoton excitation configuration at $215 \mathrm{~nm}$, by matching pulses of atoms [3] with the laser pulses to achieve optimal overlap. 
(a)

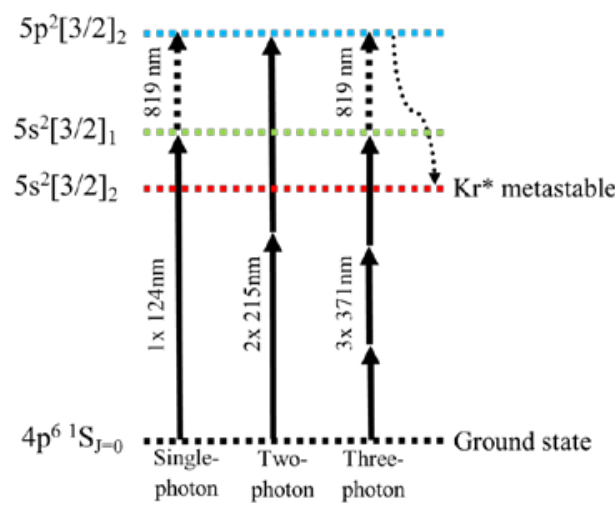

(b)

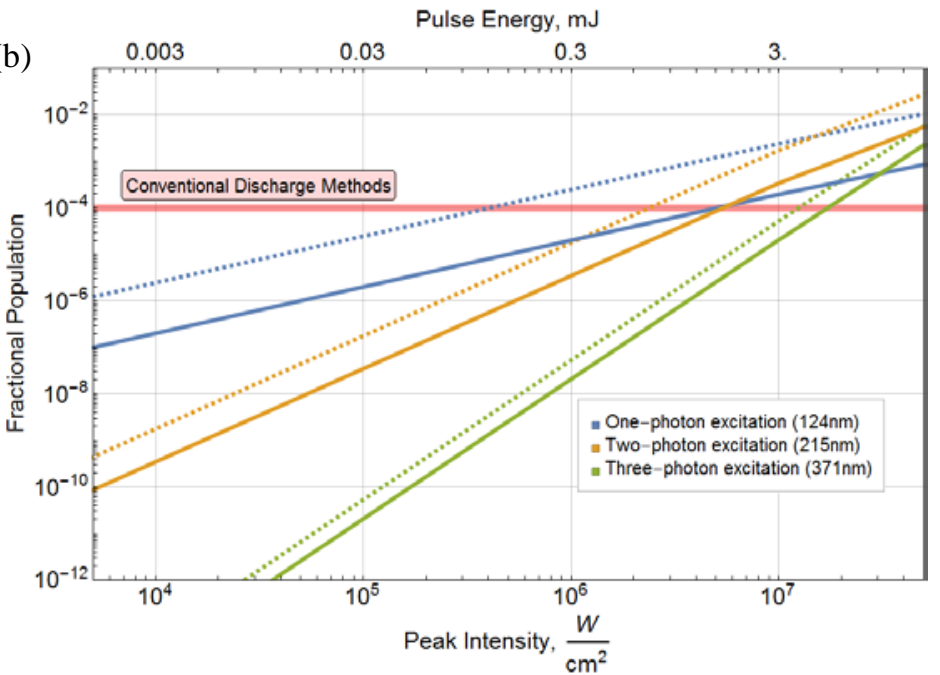

Fig. 1: (a) Energy level diagram for krypton; (b) Metastable state fractional population for single-, two- and three-photon excitation as a function of pulse energy. Solid lines: modelled using room temperature Doppler-broadening; dashed lines: modelled using reduced Doppler-broadening as achieved through transverse excitation of supersonic atom jet.

We will present comparison of modelled metastable excitation efficiencies with experimental results obtained using a broad linewidth optical parametric oscillator (OPO) for each excitation scheme.

\section{Frustrated Tunnel Ionization}

A second metastable excitation method we are considering is the strong-field excitation technique of frustrated tunnel ionization [4,5], which takes advantage of a neutral exit channel available in the tunneling regime of strongfield ionization.

By application of static electric fields, we are currently attempting to optimize excitation into the desired metastable state, rather that the Rydberg states that have previously been targeted for other applications.

We will present our measured metastable efficiencies obtained using frustrated tunnel ionization, and compare this technique with both the direct laser excitation discussed above, and with the conventional RF discharge technique currently used in ATTA systems.

\section{References}

[1] Z. T. Lu et al., "Tracer applications of noble gas radionuclides in the geosciences," Earth-Science Rev., 138 192-214 (2014)

[2] H. Daerr, "A novel vacuum ultra violet lamp for metastable rare gas experiments," Rev. Sci. Instrum. 82073106 (2011)

[3] U. Even, "The Even-Lavie valve as a source for high intensity supersonic beam,” EPJ Techn. Instrum. 217 (2015)

[4] T. Nubbemeyer et al., "Strong-Field Tunneling without Ionization,” Phys. Rev. Lett. 101233001 (2008)

[5] H. Zimmermann et al., "Strong-Field Excitation of Helium: Bound State Distribution and Spin Effects," Phys. Rev. Lett. 114123003 (2015) 